\title{
Cohesive properties of $\mathrm{CeN}$ and LaN from first principles
}

\author{
Elena Voloshina ${ }^{a *}$ and Beate Paulus ${ }^{b \dagger}$ \\ ${ }^{a}$ Max-Planck-Institut für Physik komplexer Systeme, Nöthnitzer Straße 38, 01187 Dresden, Germany \\ ${ }^{b}$ Physikalische und Theoretische Chemie, Freie Universität Berlin, Takustraße 3, 14195 Berlin, Germany
}

(Dated: December 20, 2007)

\begin{abstract}
The electron-correlation effects on the ground-state properties of CeN and LaN are studied by $a b$ initio quantum-chemical methods. The approach which is used consists in the combination of two separate steps: 1) the ground-state Hartree-Fock calculations for the crystal; 2) application of the method of increments to the studied system, which allows an expansion of bulk properties using the information from quantum-chemical calculations performed for finite cluster. As it can be expected, for CeN correlation plays a significant role: with Hartree-Fock method only $49 \%$ of the experimental cohesive energy has been covered, whereas after correlation corrections (coupled-cluster approach) the ground-state properties were found to be in good agreement with the experimental data found in literature. Thus, we obtained about $90 \%$ of the expected cohesive energy; the computed lattice constants and bulk moduli agree also well with the experimental values. For comparison the equivalent treatment has been performed for LaN, where no $f$ orbital is occupied. There the HF contribution to the ground-state properties is larger and hence the correlation effects weaker.
\end{abstract}

Key words: Ab initio calculations, Total energy and cohesive energy calculations, Electron correlation calculations, Incremental scheme, Cerium nitride, Lanthanum nitride

\section{INTRODUCTION}

Solids including lanthanides are often investigated concerning their heavy-fermion behavior, magnetic properties or the Kondo effect. These properties emerge at low temperatures and have very small energy scales, which are difficult to be described with ab initio methods. On the other hand the ground-state properties of rare-earth materials like cohesive energy, lattice structures and bulk modulus are important for the use of these systems in material science. There reliable theoretical results are desirable to understand the mechanism of binding in these materials and especially the influence of electronic correlations. For this case the magnetic structure originated from the partially filled $f$-level plays a minor role.

Cerium nitride was previously classified to be an archetypal mixed valence compound [1-4], however, recent theoretical studies on this compound performed by A. Delin et al. show that the two valence configurations (trivalent $4 f^{1}[5 d 6 s 6 p]^{3}$ and tetravalent $4 f^{0}[5 d 6 s 6 p]^{4}$ ) are far from being degenerate, and mixed valency in the ground state of $\mathrm{CeN}$ is thus highly unlikely [5]. In addition the experimental data obtained by means of X-ray absorption spectroscopy for highly pure cerium nitride [6] are also in contradiction with earlier conclusions.

For materials where the $f$-occupation is fixed, it is possible to treat the open $f$-shell with a pseudopotential. Dolg et al. have developed such pseudopotentials for the all lanthanides [7, 8] and yield very good agreement with experimental data for atoms and molecules. The application of these pseudopotentials in solids has

\footnotetext{
*Electronic address: velena@mpipks-dresden.mpg.de

$\dagger$ Electronic address: beate@chemie.fu-berlin.de
}

started with a correlated treatment of $\mathrm{GdN}[9]$. It was extended on the mean-field level to the series $\mathrm{R}_{2} \mathrm{O}_{3}(\mathrm{R}=\mathrm{La}-$ $\mathrm{Pm}$ ), where gaussian type basis sets have been optimized for the solid [10]. These studies show, that it is possible to treat the open $f$-shell with a pseudopotential on the Hartree-Fock (HF) level and including the electronic correlations with an a posteriori correlation functional. The correlation functional improves the agreement with the experiment, but the choice of the functional is somewhat arbitrary and a systematical improvement of the approach is difficult. Therefore it is desirable to include the correlations with the many-body wavefunction, using so-called quantum-chemical methods for the correlation like coupled cluster. For that reason the method of increments was developed [11] and applied to a wide range of materials including insulating rare-earth compounds like GdN [9] and $\mathrm{CeO}_{2}$ [12]. We extend these studies while applying the method of increments with a coupled-cluster treatment of the electronic correlations to CeN and LaN.

The paper is organized as follows. In the next section we introduce briefly the method of increments. The obtained results are presented in the next section (Sec. III): In Sec. III A the HF data for CeN and LaN are discussed. Sec. III B is dedicated to the correlation treatment with the incremental scheme. The calculated thermodynamic as well as mechanical properties of $\mathrm{CeN}$ and LaN are presented in Sec. III C. The obtained results of this work are compared with the ones of GdN in Sec. IIID. Conclusion follows in Sec. IV.

\section{METHOD OF INCREMENTS}

Quantum-chemical correlation methods, developed for finite systems, can be applied to periodic systems using the method of increments [11]. In this approach, the 
total energy per unit cell is written as $E=E^{\mathrm{HF}}+E^{\text {corr }}$, where $E^{\mathrm{HF}}$ is the HF energy of the system per unit cell, and $E^{\text {corr }}$ is the contribution of correlation effects to the total energy per unit cell. The correlation contribution is computed using the incremental expansion:

$E^{\mathrm{corr}}=\sum_{A} \varepsilon_{A}+\frac{1}{2 !} \sum_{A \neq B} \Delta \varepsilon_{A B}+\frac{1}{3 !} \sum_{A \neq B \neq C} \Delta \varepsilon_{A B C}+\ldots$,

where the summation over $A$ involves orbitals located in the unit cell, while those over $B$ and $C$ include all centers of the crystal. The one-body increment $\varepsilon_{A}$ is computed by considering excitations only from the $A$-orbitals, freezing the rest of the solid at the HF level. The two-body increment is defined as $\Delta \varepsilon_{A B}=\varepsilon_{A B}-\left[\varepsilon_{A}+\varepsilon_{B}\right]$, where $\varepsilon_{A B}$ is the correlation energy of the joint orbital system $A B$. Higher-order increments are defined in an analogous way. So, for three-body term we get: $\Delta \varepsilon_{A B C}=$ $\varepsilon_{A B C}-\left[\varepsilon_{A}+\varepsilon_{B}+\varepsilon_{C}\right]-\left[\Delta \varepsilon_{A B}+\Delta \varepsilon_{B C}+\Delta \varepsilon_{A C}\right]$. Finally, summing up all increments, with the proper weight factors (according to their occurrence in the solid), one obtains the exact correlation energy per unit cell of the infinite system. In order to get reliable results a sizeextensive correlation method must be used. Of course, the expansion only makes sense if the incremental expansion is well convergent, i.e., if $\Delta \varepsilon_{A B}$ rapidly decreases with increasing distance between the positions $A$ and $B$ and if the three-body terms are significantly smaller than the two-body ones. This means that only a few increments need to be calculated, yet a full account of the short range correlations is achieved this way.

\section{RESULTS AND DISCUSSION}

\section{A. Hartree-Fock calculations}

As starting point for the systematic inclusion of electron-correlation effects a reliable HF self-consistent field (SCF) treatment for the periodic system is necessary. To obtain the HF ground-state properties for solid $\mathrm{CeN}$ and $\mathrm{LaN}$ we performed CRYSTAL 06calculations [13]. For the lanthanoid we use the pseudopotentials derived by Dolg et al. for trivalent cerium and lanthane $\left[\mathrm{Ce}^{11+}\right.$-PP $\left(4 f^{1}\right.$-subconfiguration) and $\mathrm{La}^{11+}$-PP $\left(4 f^{0}\right.$-subconfiguration)] [7], thus the semicore $5 s$ - and $5 p$-orbitals were explicitly treated in the SCF procedure. For application in the CRYSTAL-calculations the basis set has to be modified (optimized by changing the exponents of the Gaussian): very diffuse exponents which are necessary to properly describe the tails of the free-atom wavefunctions cause numerical problems in CRYSTAL. For Ce as well as for La we have applied $(6 s 5 p 4 d) /[4 s 3 p 2 d]$ valence basis sets [14]. For the nitrogen atoms, the standard $6-311 \mathrm{G}^{*}$ Gaussian function basis set was used without any modification [15]. The integral tolerances were employed to compute the infinite Coulomb and HF exchange series: $10^{-7}$ for the Coulomb
TABLE I: Lattice constant $(a)$, bulk modulus $(B)$ and cohesive energy $\left(E_{\mathrm{coh}}\right)$ of $\mathrm{CeN}$ and LaN.

\begin{tabular}{lccr}
\hline \hline & $a(\AA)$ & \multicolumn{1}{c}{$B(\mathrm{GPa})$} & $E_{\mathrm{coh}}(\mathrm{eV})$ \\
\hline \multirow{3}{*}{ Experiment } & 5.01 & \multicolumn{3}{c}{ Cerium nitride $(\mathrm{CeN})$} \\
$\mathrm{nF}$ & 5.35 & 145 & -12.81 \\
$\mathrm{HF}+$ corr. & 5.14 & \multicolumn{3}{c}{109} & -11.20 \\
\hline \multirow{3}{*}{ Experiment } & 5.31 & 113 & -10.93 \\
$\mathrm{HF}$ & 5.39 & 152 & -6.79 \\
$\mathrm{HF}+$ corr. & 5.23 & 93 & -11.02 \\
\hline \hline
\end{tabular}

overlap, Coulomb penetration, HF exchange overlap, and the first exchange pseudo-overlap; $10^{-25}$ for the second exchange pseudo-overlap. The Fock matrix has been diagonalized at $72 \mathrm{k}$-points within the irreducible Brillouin zone, that corresponds to a shrinking factor of 12 in the Monkhorst net.

For evaluating cohesive energies, we subtracted the ground-state energies of the free atom. In order to achieve the same quality of the basis set for the crystal and the free atom we correct the basis set superposition error [16] via the counterpoise method [17]. In the atomic energy calculations the crystal-optimized basis set was used for the atom and also placed on the atoms of the first and second coordination shell (i.e. the corresponding basis set was supplied at the position of these atoms, but no nuclear charge or electrons were supplied). The quantum-chemical ab initio program system MOLPRO 2006 [18] was used for these calculations. At the HF level we reached $49 \%$ of the experimental cohesive energy [19] for CeN (Tab. I). This value is much lower as the percentage in $\operatorname{GdN}(63 \%[9]), \mathrm{Ce}_{2} \mathrm{O}_{3}(71 \%$ [10]), $\mathrm{CeO}_{2}(59 \%[12])$, and $\mathrm{LaN}(62 \%)$.

To calculate the lattice constant and bulk modulus we performed a fourth-order polynomial fit to the HF ground-state energy evaluated from $-2 \%$ to $+16 \%$ of the experimental lattice parameter. The HF ground-state equilibrium lattice constant $a$ was found to be $5.35 \AA$ for $\mathrm{CeN}$, that is by $7 \%$ higher than the experimental value $(5.007 \pm 0.005 \AA$ at $4.2 \mathrm{~K}[20])$. For LaN the HF equilibrium lattice constant is only $1.5 \%$ larger than the measured one [21].

The bulk modulus, $B$, is a material property that relates the change in volume with a change in pressure: $B=-V(\partial P / \partial V)_{T}$. The experimental bulk modulus can be evaluated using Ref. [22], where the data describing lattice spacings of different lanthanide nitrides as a function of pressure at room temperature has been presented. From theory, the bulk modulus can be calculated by distorting all of the dimensions of the unit cell and calculating the energy as a function of the change in volume. This procedure uses the fact that the pressure is: $P=-(\partial E / \partial V)_{T}$ and so $B=-V\left(\partial^{2} E / \partial V^{2}\right)_{T}$. The bulk modulus of cubic structure, where $V=a^{3}$, can be 
determined according to the formula

$$
B=\left(\frac{4}{9 a} \frac{\partial^{2}}{\partial a^{2}}-\frac{8}{9 a^{2}} \frac{\partial}{\partial a}\right) E(a)
$$

The $\mathrm{HF}$ value calculated at the equilibrium lattice constant is equal to $145 \mathrm{GPa}$ for $\mathrm{CeN}$, i.e. overestimates $B^{\exp }$ by about $40 \%$, and $152 \mathrm{GPa}$ for LaN, i.e. overestimates the experimental value by about $35 \%$.

\section{B. Correlation treatment}

As was mentioned above, due to the local character of the electron-correlations it is possible to determine the energy increments in finite fragments which reflect the symmetry of the crystal. This means, that in order to get reliable result one should try to include as many "crystal features" as possible and it sounds reasonable to build a cluster of crystallographic unit cell, maintaining thereby neutrality of the system. Since CeN is known to exist in sodium chloride structure, the natural choice is $\mathrm{Ce}_{4} \mathrm{~N}_{4}$-cluster. We decided to double this unit as shown in Fig. 1 (largest spheres) in order to get better description for at least two ceriums and two nitrogens. This allows us to get proper value for nearest-neighbour $\mathrm{Ce}-\mathrm{N}$ increment, which expected to be the most important for the binding in cerium nitride, as well as to describe better the nearest-neighbor $\mathrm{N}-\mathrm{N}$ and the nearest-neighbor Ce-Ce interactions. To simulate the Pauli repulsion negatively charged $\mathrm{N}$-ions are embedded with $\mathrm{Ce}^{11+}-\mathrm{PPs}$ together with corresponding minimal basis set [7] (the dark spheres of middle size in Fig. 1). Finally, the system is embedded in a set of point charges $( \pm 3$ in the interior and reduced by factor of 2,4 , and 8 at the surface planes, edges, and corners, respectively). The description of explicitly treated ions is as follows: We used for cerium $\mathrm{Ce}^{11+}$-PP together with the corresponding basis set, augmented by two $f$ - (0.67 and 0.21$)$ and one $g$ - (0.79) exponents. Nitrogen was described with ccpVTZ (Basis A) or aug-cc-pVTZ (Basis B) basis sets by Dunning [23]. The correlation energies were evaluated using the coupled cluster approach with single and double excitations and perturbative treatment of the triples [CCSD $(\mathrm{T})][24,25]$ as implemented in MOLPRO [18]. Localized orbitals used in these calculations are obtained according to the Pipek-Mezey criterion [26]. The analogous correlation calculations were performed also for LaN, applying $\mathrm{La}^{11+}-\mathrm{PP}$ and a corresponding basis set, augmented by two $f$ - (0.67 and 0.21$)$ and one $g$ - (0.79) exponents.

The calculated correlation energies for one- and twobody increments at the experimental lattice parameter are given in Tab. II. It turns out, that $\mathrm{R}-\mathrm{N}(\mathrm{R}=\mathrm{La}$ or $\mathrm{Ce}$ ) increments are much more important than $\mathrm{N}-\mathrm{N}$ increments, while R-R increments are negligibly small. For all three series $\Delta \varepsilon_{A B}$ rapidly decrease with increasing interionic distance.

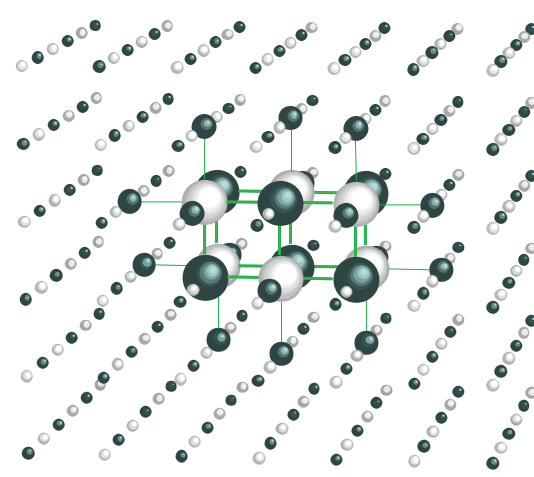

$\mathrm{Ce}^{3+}$ treated with $\mathrm{Ce}^{11+}-\mathrm{PP}$
and extended basis set
$\mathrm{Ce}^{3+}$ treated with $\mathrm{Ce}^{11+}-\mathrm{PP}$
and minimal basis set

$\mathrm{N}^{3-}$ ions

- positive (dark) and negative (light) point charges

FIG. 1: (Color online) Embedding of the $\mathrm{Ce}_{6} \mathrm{~N}_{6}$-cluster. Only the nearest neighbor point charges are shown.

TABLE II: One- and two-body correlation-energy increments (in eV) obtained with $\operatorname{CCSD}(\mathrm{T})$ method.

\begin{tabular}{|c|c|c|c|c|c|}
\hline \multirow{2}{*}{$\begin{array}{l}\text { Compound } \\
\text { Basis }\end{array}$} & \multicolumn{2}{|c|}{$\mathrm{CeN}$} & \multirow{2}{*}{$\begin{array}{c}\mathrm{LaN} \\
\mathrm{A}\end{array}$} & \multirow{2}{*}{$\begin{array}{l}\text { Weight } \\
\text { factor }\end{array}$} & \multirow[t]{2}{*}{ Dist. $^{\circ}$} \\
\hline & A & B & & & \\
\hline \multicolumn{6}{|c|}{ One-body increments $\left(\varepsilon_{\mathrm{A}}\right)$} \\
\hline$\overline{\mathrm{R}}$ & -4.9071 & -4.9460 & -4.86881 & & \\
\hline $\mathrm{N}$ & -6.7258 & -7.4429 & -6.86091 & & \\
\hline$\sum \varepsilon_{A}$ & -11.6329 & -12.3889 & -11.7297 & & \\
\hline$\sum \Delta \varepsilon_{A}$ & -0.7394 & -1.4706 & -0.9181 & & \\
\hline \multicolumn{6}{|c|}{ Two-body increments $\left(\Delta \varepsilon_{\mathrm{AB}}\right)$} \\
\hline$\overline{\mathrm{R}-\mathrm{N}}$ & -0.4150 & -0.4271 & -0.3572 & 6 & $1 / 2$ \\
\hline $\mathrm{R}-\mathrm{N}$ & -0.0154 & & -0.0114 & 8 & $\sqrt{3} / 2$ \\
\hline $\mathrm{R}-\mathrm{N}$ & -0.0054 & & -0.0034 & 24 & $\sqrt{5} / 2$ \\
\hline $\mathrm{N}-\mathrm{N}$ & -0.1326 & -0.1693 & -0.1179 & 6 & $\sqrt{2} / 2$ \\
\hline $\mathrm{N}-\mathrm{N}$ & -0.0685 & & -0.0393 & 3 & 1 \\
\hline $\mathrm{N}-\mathrm{N}$ & -0.0117 & & -0.0048 & 12 & $\sqrt{6} / 2$ \\
\hline $\mathrm{R}-\mathrm{R}$ & -0.0209 & -0.0215 & -0.0160 & 6 & $\sqrt{2} / 2$ \\
\hline $\mathrm{R}-\mathrm{R}$ & -0.0057 & & -0.0042 & 3 & 1 \\
\hline $\mathrm{R}-\mathrm{R}$ & -0.0007 & & -0.0005 & 12 & $\sqrt{6} / 2$ \\
\hline$\sum \Delta \varepsilon_{A B}$ & -4.0346 & & -3.3153 & & \\
\hline
\end{tabular}

${ }^{a}$ All distances are given in units of $a$.

TABLE III: Three-body correlation-energy increments (in $\mathrm{eV}$ ) obtained for $\mathrm{CeN}$ with $\mathrm{CCSD}(\mathrm{T})$ method.

\begin{tabular}{lccccc}
\hline \hline & \multicolumn{3}{c}{$\begin{array}{c}\text { Weight } \\
\text { factor }\end{array}$} & & Dist. $^{a}$ \\
\hline Ce-Ce-Ce & +0.0003 & 8 & $\sqrt{2} / 2$ & $\sqrt{2} / 2$ & $\sqrt{2} / 2$ \\
Ce-Ce-Ce & -0.0149 & 12 & $\sqrt{2} / 2$ & $\sqrt{2} / 2$ & 1 \\
Ce-Ce-N & +0.0013 & 12 & $1 / 2$ & $1 / 2$ & $\sqrt{2} / 2$ \\
Ce-Ce-N & -0.0021 & 3 & $1 / 2$ & $1 / 2$ & 1 \\
Ce-N-N & +0.0047 & 12 & $1 / 2$ & $1 / 2$ & $\sqrt{2} / 2$ \\
Ce-N-N & -0.0176 & 3 & $1 / 2$ & $1 / 2$ & 1 \\
N-N-N & +0.0038 & 8 & $\sqrt{2} / 2$ & $\sqrt{2} / 2$ & $\sqrt{2} / 2$ \\
N-N-N & -0.0022 & 12 & $\sqrt{2} / 2$ & $\sqrt{2} / 2$ & 1 \\
$\sum \Delta \varepsilon_{A B C}$ & -0.1595 & & & & \\
\hline \hline
\end{tabular}

${ }^{a}$ All distances are given in units of $a$. 
In order to prove a rapid convergence of the incremental expansion we have also calculated several three-body increments for CeN only. For each type of $\Delta \varepsilon_{A B C}$ we have considered two cases: the one where the three ions form an right-angled triangle or the linear one. As can be seen from Tab. III, while right-angled triangles yield positive values, cases, where between two ions of the same kind occurs an oppositely charged one, lead to attractive contributions. This difference can be explained by the fact, that in the case of right-angled triangle we deal with three pairs of nearest neighbors, which are the highest in magnitude for each sort. Therefore the individual two-body terms in this case overestimate the correlation part and therefore the three-body increment is slightly repulsive. In linear form, there is one pair of atoms at rather long distance $(5.01 \AA)$, that yields very small correlation-energy two-body increment and finally results in attractive three-body contribution. Overall, the threebody terms are significantly smaller than the two-body species.

\section{Cohesive properties}

In Fig. 2 the various contributions to the cohesive energy of CeN are presented. One can clearly see the good convergence behavior of the incremental scheme: whereas two-body correlation-energy increments have large importance, three-body terms give nearly nothing to the total correlation energy. Summing up all correlation contributions we get $-4.9349 \mathrm{eV}$. Therefore, after improving HF-value $(-6.2612 \mathrm{eV})$ by the obtained correlation-energy increments the calculated cohesive energy is $-11.1961 \mathrm{eV}$, that is rather close to the experimental one $(-12.8050 \mathrm{eV})$.

To test the influence of the quality of the basis set on the computed cohesive energy, in Tab. II we present also data where the two central nitrogens were supplied with aug-cc-pVTZ basis set. This yields an increase in

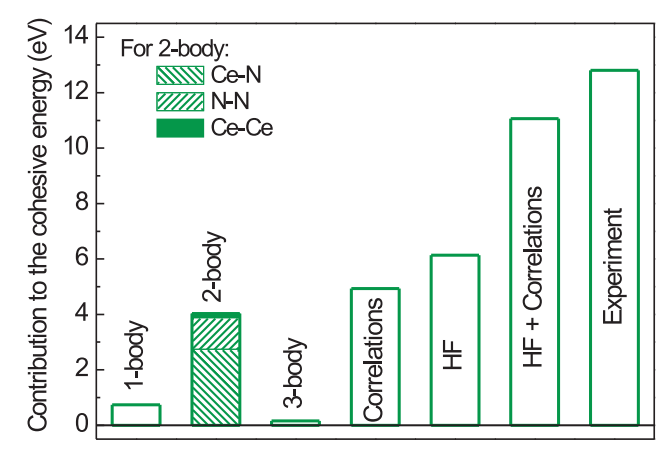

FIG. 2: (Color online) Cohesive energy of CeN: contribution to the cohesive energy of 1-, 2-, and 3-body increments and calculated cohesive energy as compared with experimental value.

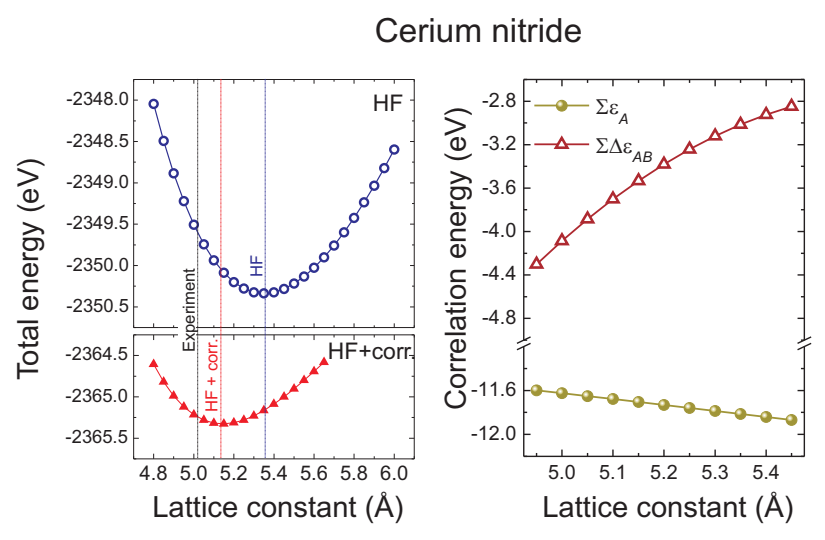

Lanthanum nitride
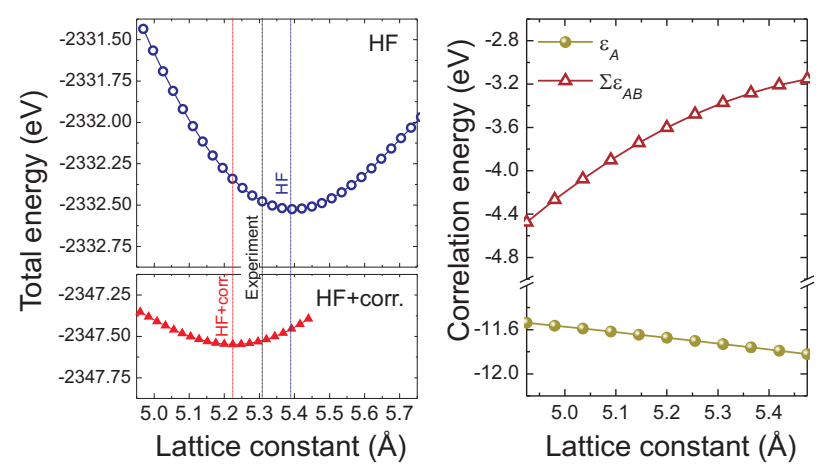

FIG. 3: (Color online) Left panel: The total energy per unit cell as a function of the lattice constant. The open circles are the data obtained at the HF level; triangles are the ones improved by inclusion of of the one- and two-body correlation-energy increments. The vertical solid line indicates the experimental lattice constant $\left(a_{\mathrm{CeN}}^{\exp }=5.01 \AA\right.$; $a_{\mathrm{LaN}}^{\exp }=5.31 \AA$ ), whereas dashed lines show the calculate ones $\left(a_{\mathrm{CeN}}^{\mathrm{HF}}=5.37 \AA\right.$ and $a_{\mathrm{CeN}}^{\mathrm{HF}+\text { corr. }}=5.14 \AA ; a_{\mathrm{LaN}}^{\mathrm{HF}}=5.39 \AA$ and $a_{\mathrm{CeN}}^{\mathrm{HF}+\text { corr. }}=5.23 \AA$ ). Right panel: Sum of the one- (closed circles) and two-body increments (triangles) as functions of lattice constant.

individual nitrogen contribution and stronger N-N interaction (the nearest neighbor $\Delta \varepsilon_{\mathrm{N}-\mathrm{N}}$ higher in magnitude by $27 \%$ for Basis B vs. Basis A); but has nearly no influence on $\Delta \varepsilon_{\mathrm{Ce}-\mathrm{N}}$, that is the highest contribution to the binding energy. As a result, we can gain about $4 \%$ with respect to the total cohesive energy.

To follow up the particular influence of increments to the mechanical properties we have presented the total energies per unit cell as functions of lattice constant obtained at HF level as well as ones improved by adding of correlation corrections. While HF lattice constant of $\mathrm{CeN}$ deviates from the experimental parameter by $7 \%$, the inclusion of the one- and two-body correlation corrections leads to a drastic shortening of the bond length (Fig. 3, left panel). The calculated value agrees with $2.5 \%$ with the measured value (Tab. I). For LaN the inclusion of correlations also shortens the lattice constant by $3 \%$ yielding a somewhat too small lattice constant compared with 
experiment. Overall it is seen, that the correlation influence is smaller in LaN than in CeN. Fig. 3 (right panel) shows sums of the one- (closed circles) and two-body increments (triangles) as a functions of the lattice constant. One can observe the same physical picture as in the case of other ionic compounds: the van-der-Waals-like interaction between the ions (included in the two-body increments) reduces the lattice constant, whereas correlations in the N-ion tend to enhance the lattice constant. The latter effect is due to the lower level spacing and therefore increasing importance of correlations at larger $a$.

Correlations effect also drastically the compresability of CeN and LaN: In both cases the bulk modulus is lowered by more than $25 \%$. For CeN the $B^{\mathrm{HF}+\text { corr }}$ is still $18 \%$ too large, whereas for LaN it is $18 \%$ too small compared to the experimental value. But the agreement with experiment is very much improved by including correlations (see Tab. I).

\section{Comparison of different lanthanide nitrides}

It seems to be very interesting to compare CeN-results with the one obtained for lanthanum nitride and GdN. These three nitrides condenses into the same crystal structure (NaCl-type) and differ only by electronic configuration of rare-earth element: $6 s^{2} 5 d^{1} 4 f^{0}, 6 s^{2} 5 d^{1} 4 f^{1}$, and $6 s^{2} 5 d^{1} 4 f^{7}$, for $\mathrm{La}, \mathrm{Ce}$, and $\mathrm{Gd}$, respectively. The data for GdN we have taken from Ref. [9], where the results are obtained for somewhat smaller basis sets, but the incremental scheme was applied with similar truncation criteria as we used. As can be seen from Fig. 4 the percentage of the experimental correlation energy to the total energy is higher in the case of CeN compared to LaN and GdN (not in the figure), where $E_{\text {corr }}^{\text {expt }}$ are nearly equal.

In all three cases cohesive contributions of one-body

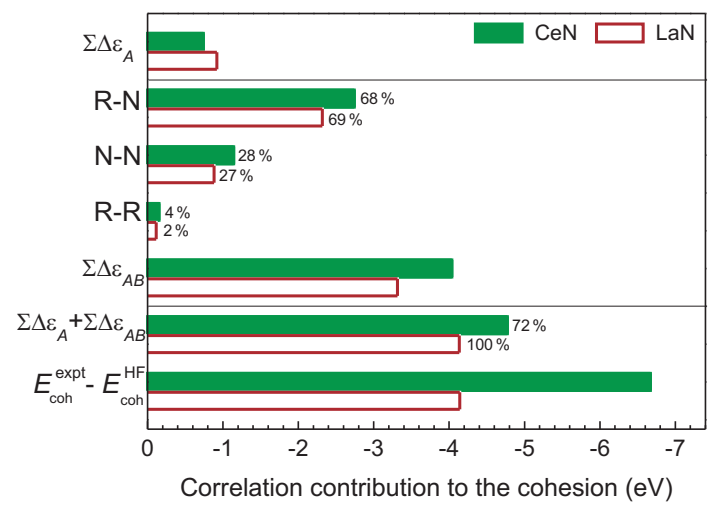

FIG. 4: (Color online) Correlation contributions to the binding energy obtained with the method of increment for CeN are plotted in comparison with LaN. increments is rather small and consists $15 \%, 20 \%$, and $25 \%$ from the sum of one- and two-body increments for $\mathrm{CeN}$, LaN, and GdN, respectively. The result obtained for the sum of two-body correlation energies related to the interaction of ions with opposite signs $\left(\sum \Delta \varepsilon_{\mathrm{RE}-\mathrm{N}}\right)$ agrees very well between each other and consist about $70 \%$. The R-R correlation-energy increments are very small in all three cases and consist about $3 \%$ of $\sum \Delta \varepsilon_{A B}$. Three-body terms for these ionic compounds are much smaller than the 2-body ones, correct the final value by $3-4 \%$, and therefore can be neglected for this comparison. Summing up one- and two-body increments one gets nearly $100 \%$ of the expected correlation energy for LaN and by about two-third for CeN, respectively. At least two reasons for such result can be found: (i) Neglection of the correlation of the $f$-shell due to the use of $4 f$-in-core $\mathrm{PP}$ yields some loss in the total correlation energy; (ii) Because of the quite large cluster size better basis set on Ce as well as $\mathrm{N}$ can not be employed. In the case of GdN taking into account estimates for the effect of a better basis set both at the one-particle level and at many-particle level, $98.5 \%$ of the experimental cohesive energy has been reached [9]. Comparison of ionic radii of $\mathrm{La}^{3+}(1.172 \AA), \mathrm{Ce}^{3+}(1.150 \AA)$, and $\mathrm{Gd}^{3+}(1.078 \AA)$ shows that the $f$-shell of Ce with only one electron is not localized as strongly as the $f$-sell in Gd. In the case of LaN with $f^{0}$ occupation of La and even in the case of $f^{7}$-subconfiguration of Gd in GdN better agreement with experiment can be achieved because of spherical symmetry of the completely empty or half-filled $f$-shell, which makes the use of an PP more reasonable. But nevertheless, the correlation treatment with a PP with an partially occupied $f$ orbital in the core improves the HF values of CeN significantly compared to experiment.

\section{CONCLUSION}

The influence of the electron correlation on the groundstate properties of the cerium nitride and lanthanum nitride were studied by $a b$ initio quantum-chemical methods. Assuming a fixed Ce valency of three [Ce(III)], a core-like treatment of the valence $f$-electron becomes possible with the pseudopotential. First, the cohesive energy, lattice constant and bulk modulus were determined at the HF level. With this method we recover only half of the experimental cohesive energy for $\mathrm{CeN}$, the computed lattice constant is overestimated by $7 \%$ and bulk modulus is a deviation of about $60 \%$ from the experimental value. As it can be expected, due to nonzero $f$-occupation of Ce correlations plays a significant role in $\mathrm{CeN}$, whereas in lanthanum nitride they are less important. As a consequence, at the HF level better agreement with experiment is found for the ground-state properties of LaN, where no f-orbitals are occupied. The correlation contributions were calculated using an expansion into local increments. After inclusion correlations obtained at the $\operatorname{CCSD}(\mathrm{T})$ level the calculated values of 
CeN are shown to be in good agreement with the experiment: we obtained about $90 \%$ of the expected cohesive energy; the computed lattice constant shows deviation of less than $3 \%$ from $a^{\text {expt }}=5.01 \AA$; bulk modulus, although improving $\mathrm{HF}$ value, overestimates $B^{\text {expt }}$ by about $18 \%$. For cohesive energy of lanthanum nitride, due to $f^{0}$-occupation of La, $100 \%$ agreement with experiment can be reached. The correlation corrected lattice constant and bulk modulus of LaN are somewhat too small compared with experiment.

\section{Acknowledgments}

We thank Professor Dr. H. Stoll (University of Stuttgart) and Professor Dr. Yu. N. Kucherenko (Institute of Metal Physics, Kiev, Ukraine) for many valuable discussions.
[1] Baer, Y.; Zürcher, C. Phys. Rev. Lett. 1977, 39956.

[2] Olcese, G. L. J. Phys. F 1979, 9, 569.

[3] Schlegel, A.; Kaldis, E.; Wachter, P.; Zürcher, C. Phys. Lett. 1978, 66B, 125.

[4] a) Kroll, P.; Eck, B.; Dronskowski, R. Adv. Mater. 2000, 12, 307; b) Landrum, G.; Dronskowski, R.; Niewa, R.; DiSalvo, F. J. Chem. Eur. J. 1999, 5, 515.

[5] Delin, A.; Oppeneer, P. M.; Brooks, M. S. S.; Kraft, T.; Wills, J. M. Phys. Rev. B 1997, 55, 10173.

[6] Kappler, J. P.; Beaurepaire, E.; Krill, G.; Serenis, J.; Godart, C.; Olcese, G. J. Phys. I France 1991, 1, 1381.

[7] Dolg, M.; Stoll, H.; Savin, A.; Preuss, H. Theor. Chim. Acta 1989, 75, 173.

[8] Dolg, M.; Fulde, P.; Küchle, W.; Neumann, C.-S.; Stoll, H. J. Chem. Phys. 1991, 94, 3011.

[9] Kaldova, S.; Dolg, M.; Flad, H.-J.; Fulde, P.; Stoll, H. Phys. Rev. B 1998, 57, 2127.

[10] Yang, J.; Dolg, M. Teor. Chem. Acc. 2005, 113, 212.

[11] Stoll, H. Phys. Rev. B. 1992, 46, 6700.

[12] Voloshina, E.; Paulus, B. J. Chem. Phys. 2006, 124, 234711.

[13] Dovesi, R.; Saunders, V. R.; Roetti, C.; Orlando, R.; Zicovich-Wilson, C. M.; Pascale, F.; Civalleri, B.; Doll, K.; Harrison, N.M.; Bush, I.J.; DArco, Ph.; Llunell, M. CRYSTAL06 Users Manual, University of Torino, Torino, 2006, see http://www.crystal.unito.it.

[14] In order to construct a basis set suitable for the periodic calculations from the basis set optimized for the Stuttgart pseudopotential $\left(\mathrm{Ce}^{11+}-\mathrm{PP}\right)$, we remove exponents below a threshold of 0.1 , and optimize the remaining most diffuse exponents. This way, the most diffuse $s-$ and $p-$ exponents are equal to 0.1 , and $d$ - exponent is 0.27 .

[15] Krishnan, R.; Binkley, J. S.; Seeger, R.; Pople, J. A. J. Chem. Phys. 1980, 72, 650.
[16] Boys, S. F.; Bernardi, F. Mol. Phys. 1970, 19, 553.

[17] van Duijneveldt, F. B.; van Duijneveldt-van de Rijdt, J. G. C. M.; van Lenthe, J. H. Chem. Rev. 1994, 94, 1873.

[18] MOLPRO, version 2006.1, a package of ab initio programs, Werner, H.-J.; Knowles, P. J.; Lindh, R.; Manby, F. R.; Schütz, M.; and others, see http://www.molpro.net.

[19] The experimental value of cohesive energy $\left(E_{c o h}^{\exp }\right)$ can be derived by appropriate Born-Haber thermodynamical cycle analysis: $E_{\text {coh }}^{\exp }=\Delta H_{\mathrm{f}}-\Delta H_{\mathrm{s}}-\frac{1}{2} \Delta H_{\mathrm{d}}$, where $\Delta H_{\mathrm{f}}$ is the standard heat of formation of solid CeN $(-340.19 \pm 6.80 \mathrm{~kJ} / \mathrm{mol}$ [Tessier, F.; Ranade, M. R.; Navrotsky, A.; Niewa, R.; DiSalvo, F. J.; Leineweber, A.; Jacobs, H. Z. anorg. und allgemeine Chemie 2001, $627,194].) ; \Delta H_{\mathrm{s}}$ stands for the heat of sublimation of the metal at $298 \mathrm{~K}(111.6 \mathrm{kcal} / \mathrm{mol}$ [Habermann, C. E.; Daane, A. H. J. Chem. Phys. 1964, 41, 2818]); and $\Delta H_{\mathrm{d}}$ $=942.0 \mathrm{~kJ} / \mathrm{mol}$ [Weast, R. C. (Editor-in-Chief); Astle, M. J.; Beyer, W. H. (Associate editors) CRC Handbook of Chemistry and Physics, 1987 (Florida: CRC Press)] is the dissociation energy of the nitrogen molecule.

[20] Danan, J.; de Novion, C.; Lallement, R. Solid State Commun. 1969, 7, 1103.

[21] Wyckoff, R. W. G. Crystal Structures, 2nd ed., 1963 (New York: Wiley), Vol. 1, p. 86.

[22] Olcese, G. L. J. Phys. F: Metal Phys. 1979, 9, 569.

[23] Dunning, Jr, T.H. J. Chem. Phys. 1989, 90, 1007.

[24] Hampel, C.; Peterson, K.; Werner, H.-J. Chem. Phys. Lett. 1992, 190, 1.

[25] Watts, J. D.; Gauss, J.; Bartlett, R. J. J. Chem. Phys., 1995, 98, 8718.

[26] Pipek, J.; Mezey, P. G. J. Chem. Phys. 1989, 90, 4916. 